\title{
Impact of Blood Pressure Cuff Inflation Rates on Flow-Mediated Dilatation and Contralateral Arm Response
}

\author{
Hsin-Fu Lin, Mandeep Dhindsa, Takashi Tarumi, Steven Miles, Daniel Umpierre, and \\ Hirofumi Tanaka
}

Department of Kinesiology and Health Education, University of Texas at Austin

Classification of First Author: Doctoral student

Abstract

Flow-mediated dilatation (FMD) is widely used as an index of nitric oxide-mediated vasodilator function, yet its methodology has not been well established. Previous research indicates that rapid inflations of a blood pressure cuff evoke systemic vasoconstriction as it was observed on non-occluded contralateral arm. This would potentially contribute to the well-known variability of FMD readings and could complicate the emerging evidence that non-occluded contralateral arm responses during the FMD procedure is associated with the presence of coronary artery disease. Purpose: To test the hypotheses that fast inflation of a blood pressure cuff could reduce FMD values and influence contralateral vasodilatory states. Method: Thirty-three apparently healthy adults (18 males and 15 females, $29 \pm 6$ years) were studied in two randomized FMD trials. The blood flow-occluding cuff was inflated fast $(<1 \mathrm{~s}$ with a Hokanson rapid cuff inflation device) in one trial or slow (over a period of 10 s) in the other during the FMD procedure. Arterial diameter, fingertip temperature, and infrared thermal images were obtained throughout each session. Results: FMD values were not different between the fast and slow cuff inflation trials ( $5.9 \pm 0.6 \%$ vs. $5.9 \pm 0.4 \%$ ). There were no differences in fingertip temperature rebound between the trials $\left(1.8 \pm 0.3\right.$ vs. $\left.1.9 \pm 0.3^{\circ} \mathrm{C}\right)$. The infrared thermal imaging revealed greater reductions in hand and finger temperature as well as more variable responses in the contralateral arm when the cuff was inflated more rapidly. Conclusion: Rates to inflate a blood pressure cuff do not appear to affect FMD readings although a fast cuff occlusion would evoke greater neurovascular changes in the non-occluded contralateral arm. 